\title{
Impurity Induced Spin Texture in Quantum Critical 2D Antiferromagnets
}

\section{Citation}

Höglund, Kaj H., Anders W. Sandvik, and Subir Sachdev. 2007. "Impurity Induced Spin Texture in Quantum Critical 2D Antiferromagnets." Physical Review Letters 98 (8). https://doi.org/10.1103/ physrevlett.98.087203.

\section{Permanent link}

http://nrs.harvard.edu/urn-3:HUL.InstRepos:41417310

\section{Terms of Use}

This article was downloaded from Harvard University's DASH repository, and is made available under the terms and conditions applicable to Other Posted Material, as set forth at http:// nrs.harvard.edu/urn-3:HUL.InstRepos:dash.current.terms-of-use\#LAA

\section{Share Your Story}

The Harvard community has made this article openly available.

Please share how this access benefits you. Submit a story.

Accessibility 


\title{
Impurity induced spin texture in quantum critical 2D antiferromagnets
}

\author{
Kaj H. Höglund, ${ }^{1}$ Anders W. Sandvik, ${ }^{2}$ and Subir Sachdev ${ }^{3}$ \\ ${ }^{1}$ Department of Physics, Åbo Akademi University, Porthansgatan 3, FI-20500, Turku, Finland \\ ${ }^{2}$ Department of Physics, Boston University, 590 Commonwealth Avenue, Boston, Massachusetts 02215 \\ ${ }^{3}$ Department of Physics, Harvard University, Cambridge, Massachusetts 02138
}

(Dated: July 3, 2018)

\begin{abstract}
We describe the uniform and staggered magnetization distributions around a vacancy in a quantum critical two-dimensional $S=1 / 2$ antiferromagnet. The distributions are delocalized across the entire sample with a universal functional form arising from an uncompensated Berry phase. The numerical results, obtained using quantum Monte Carlo simulations of the Heisenberg model on bilayer lattices with up to $\approx 10^{5}$ spins, are in good agreement with the proposed scaling structure. We determine the exponent $\eta^{\prime}=0.40 \pm 0.02$, which governs both the staggered and uniform magnetic structure away from the impurity and also controls the impurity spin dynamics.
\end{abstract}

PACS numbers: 75.10.Jm, 75.10.Nr, 75.40.Cx, 75.40.Mg

Some of the most interesting physics of strongly interacting quantum systems arises in their response to impurities. Metallic systems exhibit the Kondo effect, and much rich physics has been discovered in their response to a magnetic impurity which carries a localized spin $S$. In contrast, Mott insulators have a particularly rich response to non-magnetic impurities. For example, the spin-gap compound $\mathrm{CuGeO}_{3}$, which consists of dimerized pairs of $S=1 / 2 \mathrm{Cu}$ ions locked into $S=0$ valence bonds, acquires magnetic order upon replacing a very small density of the $\mathrm{Cu}$ with spinless $\mathrm{Zn}$ ions [1]; it is believed that an unpaired $\mathrm{Cu}$ spin is localized in the vicinity of the $\mathrm{Zn}$ impurity, and behaves like a localized $S=1 / 2$ moment. The cuprate superconductors have also seen a variety of studies [2, 3, 4, [5] of the spin and charge correlations in the vicinity of $\mathrm{Zn}$ ions replacing the $\mathrm{Cu}$ ions within a superconducting layer; here there is also an unpaired spin, but its spatial distribution and dynamics are not fully understood.

This paper will explore the effect of a non-magnetic impurity on a quantum critical Mott insulator, at the boundary between a magnetically ordered and a spin-gap state. We will describe the fate of the spatial magnetic structure of the localized impurity in the spin-gap state, as this state is tuned to the quantum critical point. We find that the strongly-interacting gapless excitations in the bulk lead to a non-trivial and universal spatial form, with power-law decay of spin correlations away from the impurity (analogous to skyrmions induced by dopants in the Néel state [6]). We will present new numerical and analytic results on the spatial spin distribution, building upon the scaling structure proposed in an earlier fieldtheoretical analysis [7, 8]. Our results are of relevance to Mott insulators which can be tuned across the quantum phase transition. They also shed light on the cuprates, which are are in the vicinity of a magnetic ordering quantum phase transition.

In our numerical investigations of a model spin-gap Mott insulator, we consider the spin- $1 / 2$ Heisenberg an- tiferromagnet on a bilayer lattice. It is defined by the Hamiltonian

$$
H=J \sum_{\langle i, j\rangle} \mathbf{S}_{1, i} \cdot \mathbf{S}_{1, j}+J_{\perp} \sum_{i} \mathbf{S}_{1, i} \cdot \mathbf{S}_{2, i},
$$

where $\mathbf{S}_{a, i}$ is a spin- $1 / 2$ operator at site $i$ on layer $a=1,2$, and $\langle i, j\rangle$ denotes a pair of nearest-neighbor sites on an $L \times L$ open-boundary square lattice. With intralayer interactions in only one of the layers (a Kondo lattice), as shown in Fig. 1, the model has a quantum critical point when the ratio $g=J_{\perp} / J$ is $g_{c}=1.3888(1)$ [9], with the spin-gap state present for $g>g_{c}$, and an antiferromagnetically ordered state for $g<g_{c}$. There is convincing evidence [9, 10] that this quantum critical point is described by the Wilson-Fisher fixed point of $\varphi^{4}$ field theory with $\mathrm{O}(3)$ symmetry in 3 spacetime dimensions. Here, our discussion is conveniently presented in terms of the fixed-length formulation of this field theory, which is the $\mathrm{O}(3)$ non-linear sigma model of the field unit length field $\mathbf{n}(r, \tau)\left(\mathbf{n}^{2}=1\right)$, representing the local orientation of the antiferromagnetic order, with action

$$
\mathcal{S}_{\text {bulk }}=\frac{1}{2 \widetilde{g}} \int d \tau \int d^{2} r\left[\left(\partial_{\tau} \mathbf{n}\right)^{2}+c^{2}\left(\nabla_{r} \mathbf{n}\right)^{2}\right],
$$

where $\tau$ is imaginary time, $r$ is the spatial co-ordinate, $c$ is the spin-wave velocity, and the coupling $\widetilde{g}$ is a monotonic function of $g$. At the quantum critical point at $\widetilde{g}=\widetilde{g}_{c}$, the correlations of the antiferromagnetic order are characterized by the power-law decay

$$
\langle\mathbf{n}(r, \tau) \cdot \mathbf{n}(0,0)\rangle \sim\left(r^{2}+c^{2} \tau^{2}\right)^{-(1+\eta) / 2},
$$

with the exponent $\eta \approx 0.04[11]$ of the Wilson-Fisher fixed point.

Let us now add a non-magnetic impurity to the above systems. For the lattice Hamiltonian $H$, we create a vacancy by removing a single spin. In a finite size $L \times L$ system, this leads to a twofold degenerate $S=1 / 2$ ground state. We take $L$ odd and place the vacancy at the center of the top layer, as shown in Fig. 1 We choose the 


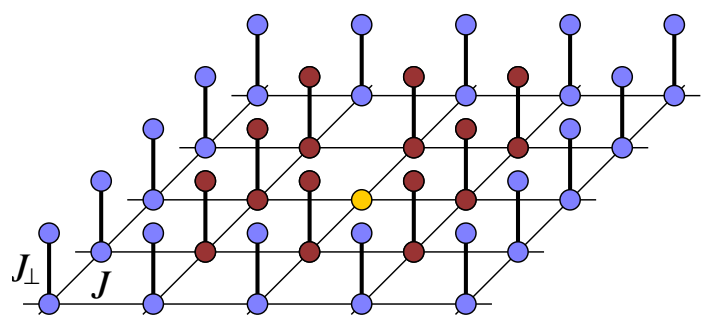

FIG. 1: (Color online) An $L=5$ incomplete bilayer model with a vacancy. The single unpaired central spin (yellow) constitutes frame $R=0$. Frames $R=1$ and $R_{\max }=2$ consist, respectively, of the surrounding red and blue sites.

ground states to be eigenstates of the total $S^{z}$; in either of these states, all the $\left\langle S^{z}{ }_{1,2, i}\right\rangle$ are non-zero even in zero applied field (which we assume throughout), and exhibit an interesting spatial structure that we will describe.

Next, we add the impurity to the field theory. The unpaired spin near the origin leads to a net uncompensated Berry phase between the antiferromagnetically oriented spins, adding an impurity term to the action [8]

$$
\mathcal{S}_{\mathrm{imp}}=i S \int d \tau \mathbf{A}[\mathbf{n}(0, \tau)] \cdot \frac{d \mathbf{n}(0, \tau)}{d \tau},
$$

which depends only upon the orientation of the order parameter at $r=0$. Here $S=1 / 2$ is the unpaired spin associated with the non-magnetic impurity, and $\mathbf{A}$ is the Dirac monopole function in spin space with $\nabla_{\mathbf{n}} \times \mathbf{A}=\mathbf{n}$. It was argued in Refs. [7, 8] that $\mathcal{S}_{\text {bulk }}+\mathcal{S}_{\text {imp }}$ universally describes the quantum impurity near $g=g_{c}$. In the infinite system, the critical spin correlations are characterized by a different "boundary" exponent at $r=0$ :

$$
\langle\mathbf{n}(0, \tau) \cdot \mathbf{n}(0,0)\rangle \sim|\tau|^{-\eta^{\prime}} .
$$

A crucial property of the field theory $\mathcal{S}_{\text {bulk }}+\mathcal{S}_{\text {imp }}$ is that, like the lattice model, for $g \geq g_{c}$ the ground state has total spin $S$. This means that for the conserved Noether magnetization density $Q_{z}(r)$, associated with the symmetry of $\mathrm{O}(3)$ rotations of the action, has a non-vanishing expectation value even in zero field, and obeys

$$
\left\langle\int d^{2} r Q_{z}(r)\right\rangle=S
$$

in the ground state with maximum spin projection in the $z$ direction. While this spin is localized in the spin-gap state with $g>g_{c}$, there is a transition to a delocalized critical state at $g=g_{c}$ in which (as we describe below) the spatial extent of the magnetization is set only by the system size. Consequently, in an infinite system at $g=g_{c}$ we have $\left\langle Q_{z}(r)\right\rangle=0$ at all $r$ even though Eq. (6) is obeyed [12].

A proper analysis of the delocalized spin texture in the ground state at $g=g_{c}$ requires imposition of a finite size $L$. We found from our numerical results, described below, that the spin distribution quite accurately obeys the scaling form

$$
\left\langle Q_{z}(r)\right\rangle=L^{-2} \Phi_{Q}(r / L),
$$

where $\Phi_{Q}(y)$ is a universal function (with no arbitrary scale factors) obeying $\int d^{2} y \Phi_{Q}(y)=S$. The same scaling form also emerges from a renormalization group analysis of the field theory $\mathcal{S}_{\text {bulk }}+\mathcal{S}_{\text {imp }}$, along with explicit results for $\Phi_{Q}(y)$, and these will be described elsewhere. More useful here is the behavior of the scaling function as $y \rightarrow 0$, which describes the spin distribution in the vicinity of the impurity. A key feature of the theory [7] is that both the uniform and staggered spin operators of the bulk theory transmute into the same "boundary" operator as they approach the impurity. Here we are using $\mathbf{n}(0, \tau)$ to represent this boundary operator, and so the scaling dimensions implicit in Eqs. (55 7) suggest the operator product expansion $\lim _{r \rightarrow 0} \mathbf{Q}(r, \tau) \sim|r|^{-2+\eta^{\prime} / 2} \mathbf{n}(0, \tau)$, which in turn implies that

$$
\Phi_{Q}(y \rightarrow 0) \sim y^{-2+\eta^{\prime} / 2} .
$$

Similarly, we can also examine the distribution of staggered spin density, which is encoded in the spatial distribution of the order parameter $\mathbf{n}$. From Eq. (3) we deduce the scaling form

$$
\left\langle n_{z}(r)\right\rangle=L^{-(1+\eta) / 2} \Phi_{n}(r / L) .
$$

Now the operator product expansion to the same boundary operator is $\lim _{r \rightarrow 0} \mathbf{n}(r, \tau) \sim|r|^{-\left(1+\eta-\eta^{\prime}\right) / 2} \mathbf{n}(0, \tau)$, and this leads to

$$
\Phi_{n}(y \rightarrow 0) \sim y^{-\left(1+\eta-\eta^{\prime}\right) / 2} .
$$

Unlike $\Phi_{Q}$, the integral of $\Phi_{n}$ is not quantized, and its overall scale is non-universal. Note that the theoretical results in Eqs. (7819/10) are tightly constrained, dependent only upon a single exponent $\eta^{\prime}$ in addition to the standard bulk critical correlation function exponent $\eta \approx 0.04$. The value of $\eta^{\prime}$ has previously been estimated in the time domain, using Eq. (5) [14]. We will show below that the numerics confirm that the spatial structure is also governed by this exponent, which we evaluate to higher precision than previously.

In order to numerically study how the impurityinduced total magnetization $S^{z}=\sum_{i} S_{i}^{z}= \pm 1 / 2$ is distributed in the system at $T=0$, the lattice is decomposed into "frames" surrounding the vacancy, as illustrated in Fig. 1. For each frame $R$ we determine the uniform and staggered magnetizations, respectively, defined by,

$$
\begin{aligned}
& M_{0}(R)=\left\langle s \sum_{i \in R}\left(S_{1, i}^{z}+S_{2, i}^{z}\right)\right\rangle, \\
& M_{\pi}(R)=\left\langle s \sum_{i \in R}(-1)^{x_{i}+y_{i}}\left(S_{1, i}^{z}-S_{2, i}^{z}\right)\right\rangle,
\end{aligned}
$$




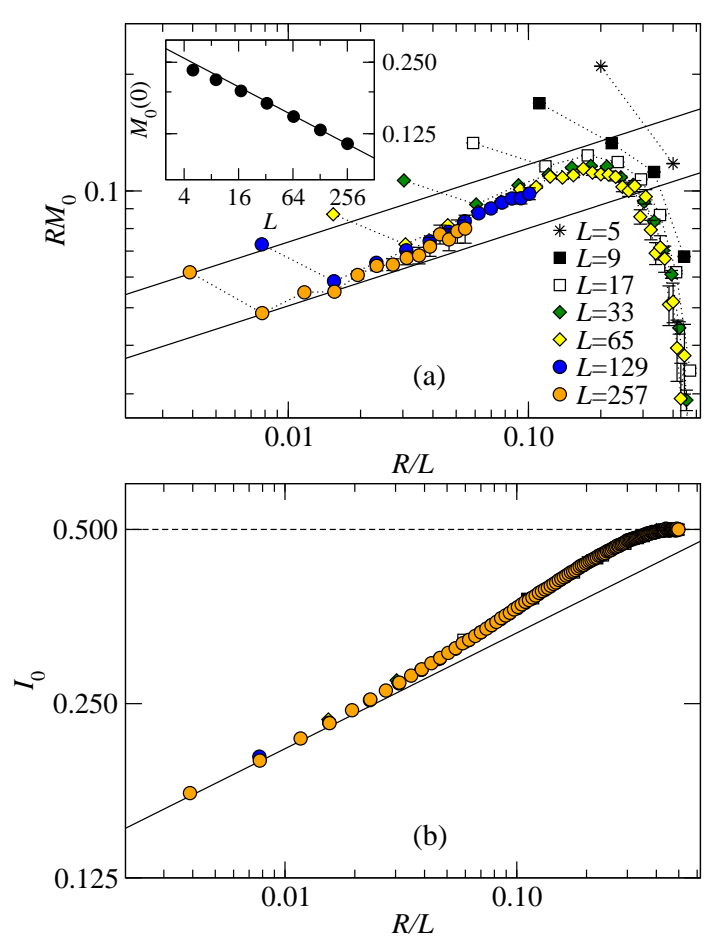

FIG. 2: The uniform frame magnetization $M_{0}(R)$ (a) and the corresponding integrated quantity $I_{0}(R)$ (b) plotted according to the predicted scaling laws for several system sizes. In the inset of (a) we show the magnetization at $R=0$, from which we obtain the exponent $\eta^{\prime}=0.40 \pm 0.02$. All other lines are shown using this value for the exponent. In (b) the data collapse is so tight that the $L=257$ points almost completely hide the data for smaller $L$.

where $s=2 S^{z}= \pm 1$ is included in order to make the contributions positive for both $S^{z}= \pm 1 / 2$ states. Expectation values are calculated in $T>0$ quantum Monte Carlo simulations utilizing the stochastic series expansion algorithm [15]. Ground state results are obtained by choosing a sufficiently low temperature for each $L$. For the largest lattice we have studeied, $L=257$, temperatures as low as $T / J \approx 10^{-3}$ are required for satisfactory $T \rightarrow 0$ convergence [16].

The uniform frame magnetization is related to the magnetization density $Q_{z}$ by $M_{0}(R) \propto R Q_{z}(R)$ for $R>0$. Hence, according to the scaling forms (78), we should have for $R / L$ small

$$
\begin{aligned}
M_{0}(R) & \sim \frac{1}{R}\left(\frac{R}{L}\right)^{\eta^{\prime} / 2} \\
I_{0}(R) & \sim\left(\frac{R}{L}\right)^{\eta^{\prime} / 2}
\end{aligned}
$$

where $I_{0}(R)$ is the integrated frame magnetization,

$$
I_{0}(R)=\sum_{r=0}^{R} M_{0}(r),
$$

which has to be exactly $1 / 2$ at the edge of the lattice, i.e., for $R_{\max }=(L-1) / 2$. The uniform magnetization results are shown versus $R / L$ in log-log plots in Fig. 2. The upper panel shows the frame magnetization, which is seen to collapse onto a single curve for $L \gtrsim 17$, with the exception of the $R=1$ points which scale with a different prefactor. Power-law behavior is seen for small $R / L$, including also the $R=1$ data which approach such behavior for larger $L$. For a fixed $R, I_{0}(R)$ decays according to Eq. (14) as $L^{-\eta^{\prime} / 2}$. This should hold also for $R=0$, because $I_{0}\left(R_{\max }\right)=1 / 2$. The inset of the upper panel of Fig. 2 shows the scaling of the $R=0$ magnetization, which in fact gives us the most precise determination of the exponent; $\eta^{\prime}=0.40 \pm 0.02$. We use this value of $\eta^{\prime}$ to draw the lines shown in the main panels of Fig. 2. The integrated magnetization $I_{0}(R)$, shown in the lower panel, scales even better than $M_{0}$, with also data for the smallest lattices falling on the same curve.

The theory is expected to capture accurately the behavior for large lattices, far from the impurity and the edges, i.e., for large $R$ but small $R / L$. It is therefore quite remarkable that even the $M_{0}(R=2)$ data fall on the common scaling curve and that the integrated magnetization scales almost perfectly even for very small lattices. The asymptotic power-law behavior is closely approached below $R / L \approx 0.02$.

Next we consider the staggered component (12) of the impurity induced texture and the corresponding integral

$$
I_{\pi}(R)=\sum_{r=0}^{R} M_{\pi}(r) .
$$

Eqs. (9110) imply for small $R / L$

$$
\begin{aligned}
M_{\pi}(R) L^{-(1-\eta) / 2} & \sim\left(\frac{R}{L}\right)^{\left(1+\eta^{\prime}-\eta\right) / 2}, \\
I_{\pi}(R) L^{-(3-\eta) / 2} & \sim\left(\frac{R}{L}\right)^{\left(3+\eta^{\prime}-\eta\right) / 2} .
\end{aligned}
$$

Results are shown in Figs. 3, We observe good data collapse of $M_{\pi}$ for all $L$, and the slope vs $R / L$ agrees very well with the value $\eta^{\prime}=0.40$ determined above from the uniform magnetization. In this case the integrated quantity shows substantial subleading size corrections for small $R$, but an $L \rightarrow \infty$ approach to the power-law behavior shown by the line appears very plausible.

In conclusion, we have presented analytical and numerical results for the uniform and staggered components of the spin texture induced by a static vacancy in a 2D quantum antiferromagnet at its quantum critical point. The theory predicts scaling functions with asymptotic power-law behaviors, which are very well reproduced by the numerics. We have determined the value $\eta^{\prime}=0.40 \pm 0.02$ for the single exponent governing the asymptotic behavior of both the uniform and staggered structure. This exponent characterizes the influence of 

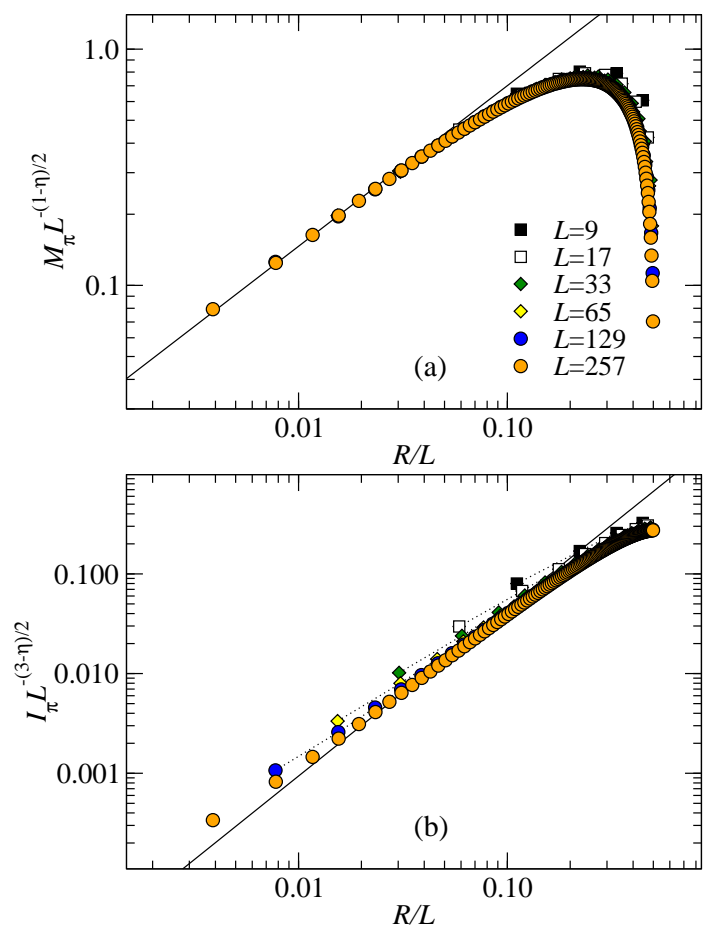

FIG. 3: Size and distance scaling of the staggered frame magnetization $M_{\pi}(R)$ (a) and the corresponding integrated quantity $I_{\pi}(R)(\mathrm{b})$. The over-all $L$ dependence, $L^{-(1-\eta) / 2}$ and $L^{-(3-\eta) / 2}$ for $M_{\pi}$ and $I_{\pi}$, respectively, has been divided out. The lines in (a) and (b) have slopes 0.68 and 1.68 , respectively, corresponding to the value of the exponent $\eta^{\prime}=0.40$ extracted from the $M_{0}(0)$ results in Fig. 2.

the Berry phase in $\mathcal{S}_{\mathrm{imp}}$ and so, unlike the bulk theory, cannot be related to the exponent of any classical theory in one higher dimension. Our results support a central property of the "boundary" critical field theory [7]: the bulk operators for the staggered and uniform magnetizations transmute into the same boundary spin operator as they approach the impurity. Earlier time domain studies [14] yielded $\eta^{\prime}=0.37 \pm 0.05[14]$; the good agreement between the two approaches provides strong evidence that a single exponent indeed governs both the temporal and spatial impurity effects [7]. In addition to extracting the asymptotic power-law, our numerical calculations also give the full scaling functions for arbitrary distance from the impurity. We note that the integrated effects of the impurity are much stronger at criticality than in the symmetry-broken Néel state, where the induced disturbance of the magnetic structure around the impurity decays asymptotically as $r^{-3}$ [17].

NMR [2, 3] and STM [4, 5] experiments have probed the magnetization distributions around an impurity. Although these systems are not at a quantum critical point, we hope similar experiments in related system will be in the quantum critical regime, allowing measurements of the exponent $\eta^{\prime}$ using some of the observables discussed in Ref. 7 .
This work was supported by the NSF under grants No. DMR-0513930 (AWS) and DMR-0537077 (SS). A travel grant awarded by the Finnish Academy of Science and Letters from the Vilho, Yrjö, and Kalle Väisälä Foundation is also gratefully acknowledged (KHH). Part of the simulations were performed at the CSC, the Finnish IT Center for Science.

[1] K. Manabe, H. Ishimoto, N. Koide, Y. Sasago, and K. Uchinokura, Phys. Rev. B 58, R575 (1998).

[2] J. Bobroff, H. Alloul, W. A. MacFarlane, P. Mendels, N. Blanchard, G. Collin, and J.-F. Marucco, Phys. Rev. Lett. 86, 4116 (2001).

[3] S. Ouazi, J. Bobroff, H. Alloul, M. Le Tacon, N. Blanchard, G. Collin, M. H. Julien, M. Horvatić, and C. Berthier, Phys. Rev. Lett. 96, 127005 (2006).

[4] A. Yazdani, B. A. Jones, C. P. Lutz, M. F. Crommie, and D. M. Eigler, Science 275, 1767 (1997).

[5] S. H. Pan, E. W. Hudson, K. M. Lang, H. Eisaki, S. Uchida, J. C. Davis, Nature (London) 403, 746 (2000); E W. Hudson, K. M. Lang, V. Madhavan, S. H. Pan, H. Eisaki, S. Uchida, and J. C. Davis, Nature (London) 411, 920 (2001).

[6] E. C. Marino, Phys. Lett. A 263446 (1999).

[7] S. Sachdev, C. Buragohain, and M. Vojta, Science 286, 2479 (1999); M. Vojta, C. Buragohain, and S. Sachdev, Phys. Rev. B 61, 15152 (2000).

[8] S. Sachdev, and M. Vojta, Phys. Rev. B 68, 064419 (2003).

[9] V. N. Kotov, O. Sushkov, Z. Weihong, and J. Oitmaa, Phys. Rev. Lett. 80, 5790 (1998); L. Wang, K. S. D. Beach, and A. W. Sandvik, Phys. Rev. B 73, 014431 (2006); W. Brenig, ibid., 73, 104450 (2006).

[10] M. Matsumoto, C. Yasuda, S. Todo, and H. Takayama, Phys. Rev. B 65, 014407 (2002).

[11] M. Campostrini, M. Hasenbusch, A. Pelissetto, P. Rossi, and E. Vicari, Phys. Rev. B 65, 144520 (2002).

[12] A related result is that despite the quantization of total spin in the ground state, the finite temperature $(T)$ response of an infinite system to a uniform applied magnetic field need not have the Curie value; the latter is the response of gapless continuum of low energy states, and not of the ground state manifold alone. The theory [7, 13] predicts a spin susceptibility $\chi=\mathcal{C} / T$, with $\mathcal{C} \neq S(S+1) / 3$. Numerical efforts to extract $\mathcal{C}$ will be discussed elsewhere (K. H. Höglund and A. W. Sandvik, cond-mat/0701472); here we focus on impurity effects at $T=0$.

[13] A. V. Syromyatnikov and S. V. Maleyev, Phys. Rev. B 74, 184433 (2006).

[14] M. Troyer, Prog. Theor. Phys. Supplement 145, 326 (2002).

[15] A. W. Sandvik, Phys. Rev. B 56, 11678 (1997); Phys. Rev. B 59, R14157 (1999).

[16] The memory and CPU time needed for these computations scale as $N / T$. At the lowest temperature, $T / J \approx$ 0.00117 , considered for the largest lattice, $L=257$, approximately $3 \mathrm{~GB}$ of RAM was required. It is currently difficult to go to lower $T$ and/or larger $L$.

[17] A. Lüscher and O. P. Sushkov, Phys. Rev. B 71, 064414 (2005). 